\title{
A SIMPLE AND FAST SEMIAUTOMATIC PROCEDURE FOR THE ATOMISTIC MODELLING OF COMPLEX DNA POLYHEDRA
}

Cassio Alves ${ }^{1 \#}$, Federico lacovelli ${ }^{2 \#}$, Mattia Falconi ${ }^{2}$, Francesca Cardamone ${ }^{2}$, Blasco Morozzo della Rocca ${ }^{2}$, Cristiano L.P. de Oliveira*3 and Alessandro Desideri*2

${ }^{1}$ Instituto de Fisica, Grupo de Fluidos Complexos, Universidade de São Paulo, Caixa Postal 66318, 05314-970

Sao Paulo, Brasil and Department of Engineering and Sciences, Federal University of Paraná, 85950-000, Palotina, Paraná, Brazil.

Department of Biology, University of Rome "Tor Vergata", Via della Ricerca Scientifica, 00133, Rome, Italy.

${ }^{3}$ Instituto de Fisica, Grupo de Fluidos Complexos, Universidade de São Paulo, Caixa Postal 66318, 05314-970 Sao Paulo, Brasil.

${ }^{*}$ Corresponding authors:

Prof. Alessandro Desideri Department of Biology, University of Rome "Tor Vergata", Via della Ricerca Scientifica 1, 00133 Rome, Italy. *E-mail: desideri@uniroma2.it, Tel. +39.06.72594376; Fax. +39.06 .2022798 .

Prof Cristiano Luis Pinto Oliveira, Instituto de Fisica, Grupo de Fluidos Complexos, Universidade de São Paulo, Caixa Postal 66318, 05314-970 Sao Paulo, Brasil. *E-mail: crislpo@if.usp.br

Author contributions ${ }^{\#}$ These authors contributed equally to this work. 


\section{Minimization procedure}

The set of coordinates for all DNAs ds is obtained minimizing the function:

$$
\chi^{2}=\sum_{i=1}^{n}\left(y_{i}-y_{\exp }\right)^{2}
$$

where $y_{\exp }$ is the distance, between the strands of two different helices, expected to accommodate the single strand linker, and $y_{i}$ is the distance obtained with the minimization. The best condition has been obtained constraining the function below a threshold, which can be set by the user (a typical condition $\chi^{2}<1$ is used in the program). The program employs a standard simulating annealing procedure, ${ }^{1,2}$ since this heuristic method is satisfactory for a fast search of the space parameters that minimizes the previously described function [1]. A temperature parameter is associated with the system in order to control the changes of the other parameters. Starting at high temperature the system is cooled, according with the number of iterations. The optimization starts from a random set of parameters, chosen inside a userdefined range and the procedure searches the conformational space to reduce $\chi^{2}$ (ref 23 and 24 - L. Ingber,1993 and Svergun, Biophys. J., 1999). The cage model is assembled, the final atomic positions are defined and the model is exported in a standard PDB file format when the value of $\chi^{2}$ is below the limit defined by user, or when the program reaches the maximum number of iterations.

\section{References}

(1) Ingber, L. Simulated Annealing: Practice versus Theory. Math. Comput. Model. 1993, 18 (11), $29-57$.

(2) Svergun, D. I. Restoring Low Resolution Structure of Biological Macromolecules from Solution Scattering Using Simulated Annealing. Biophys. J. 1999, 76 (6), 2879-2886. 


\section{Supplementary figures}
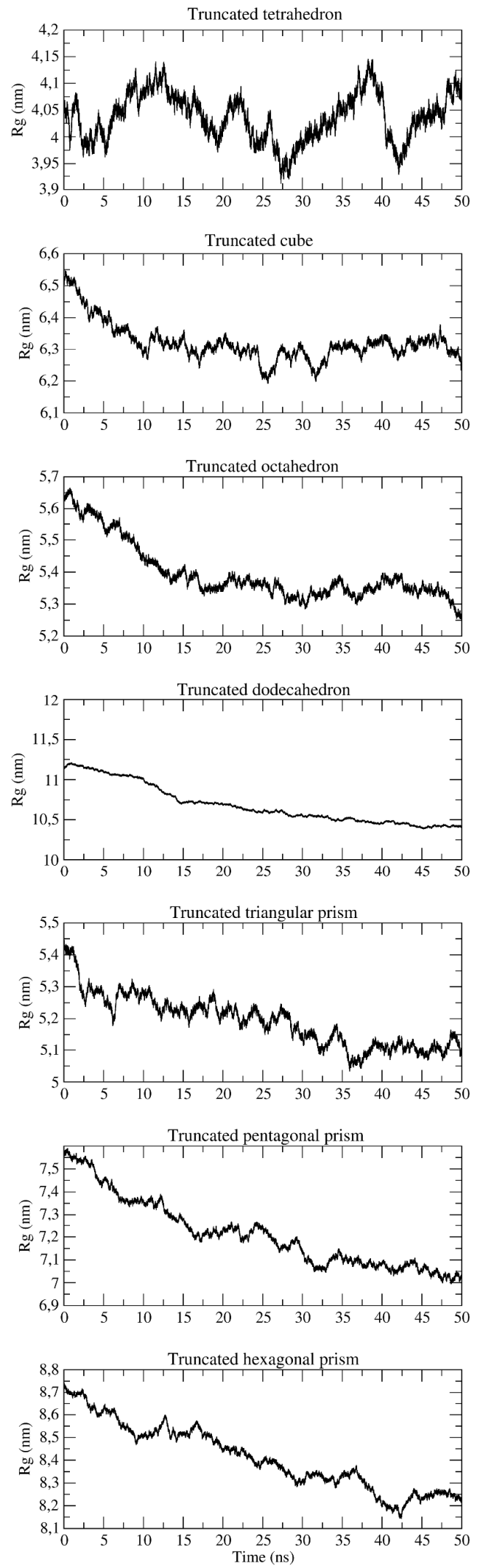

Figure S1. Gyration radius as a function of simulation time calculated for the seven modelled geometries. 


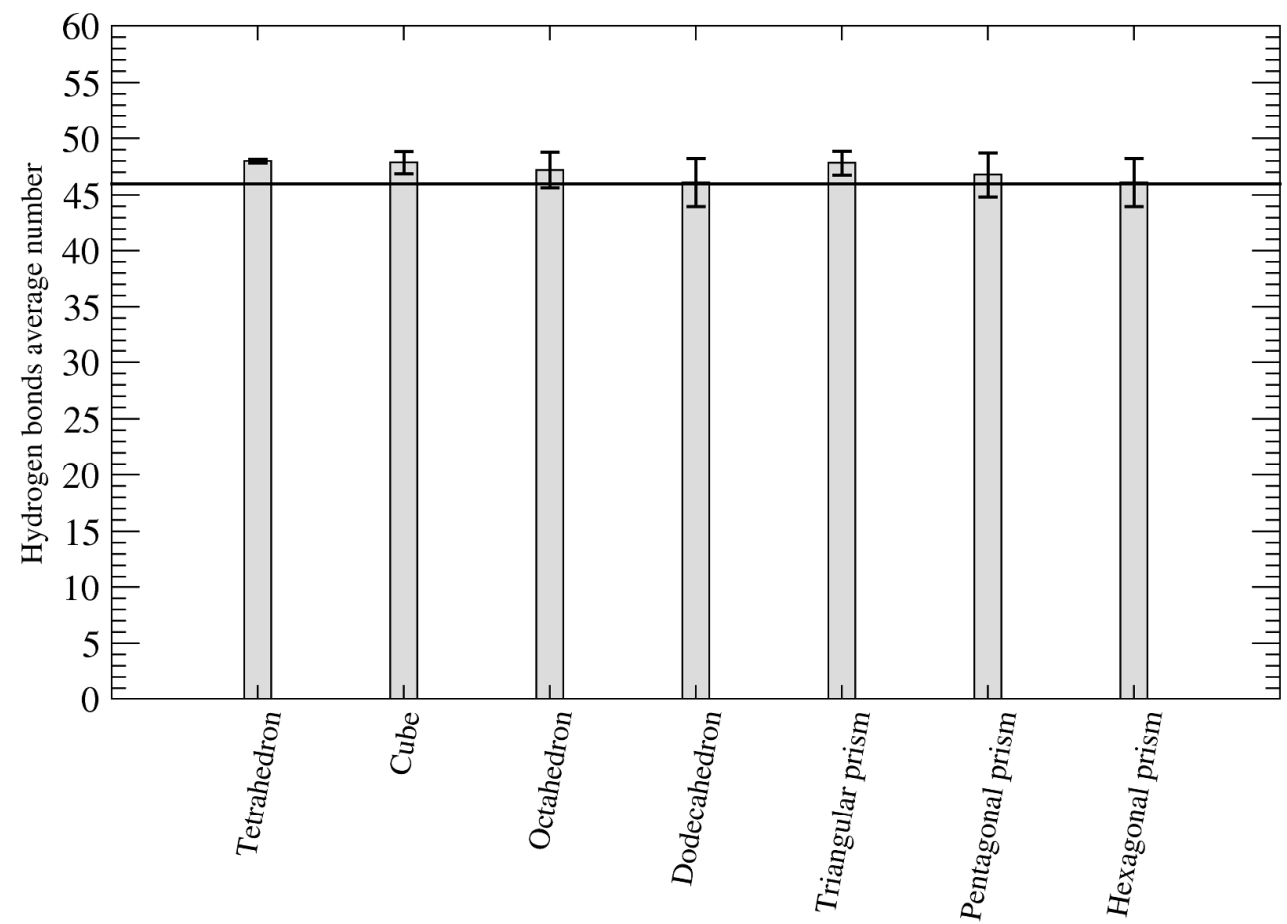

Figure S2. Histogram indicating the average number of hydrogen bonds established in the DNA double helices of the seven different cages.
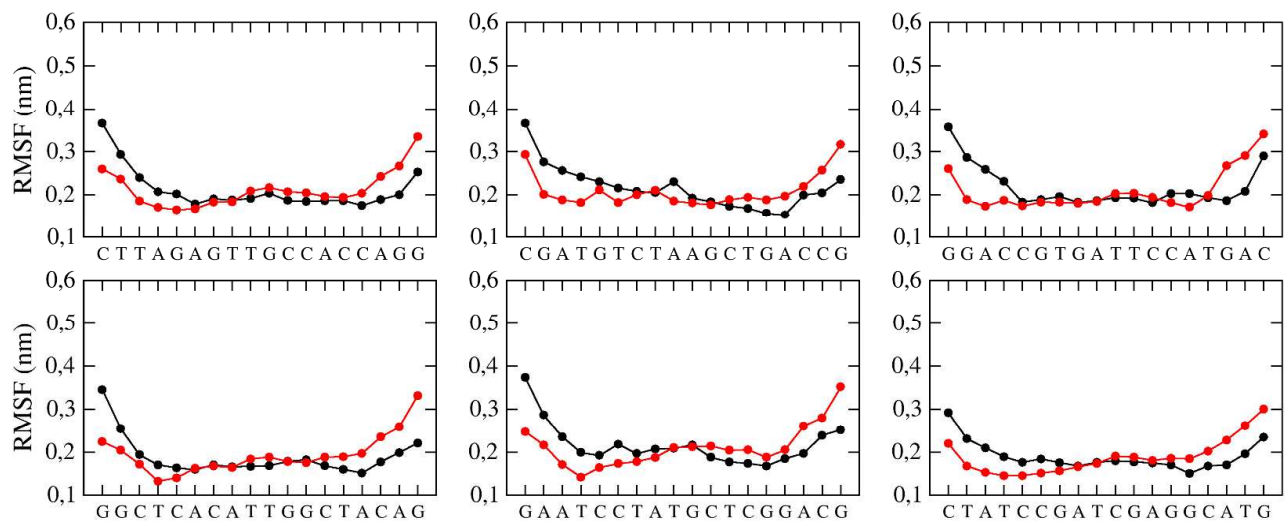

Figure S3A. Local RMSF values calculated for the double helices of the truncated tetrahedron cage. Black and red filled circles specify the two strands. 

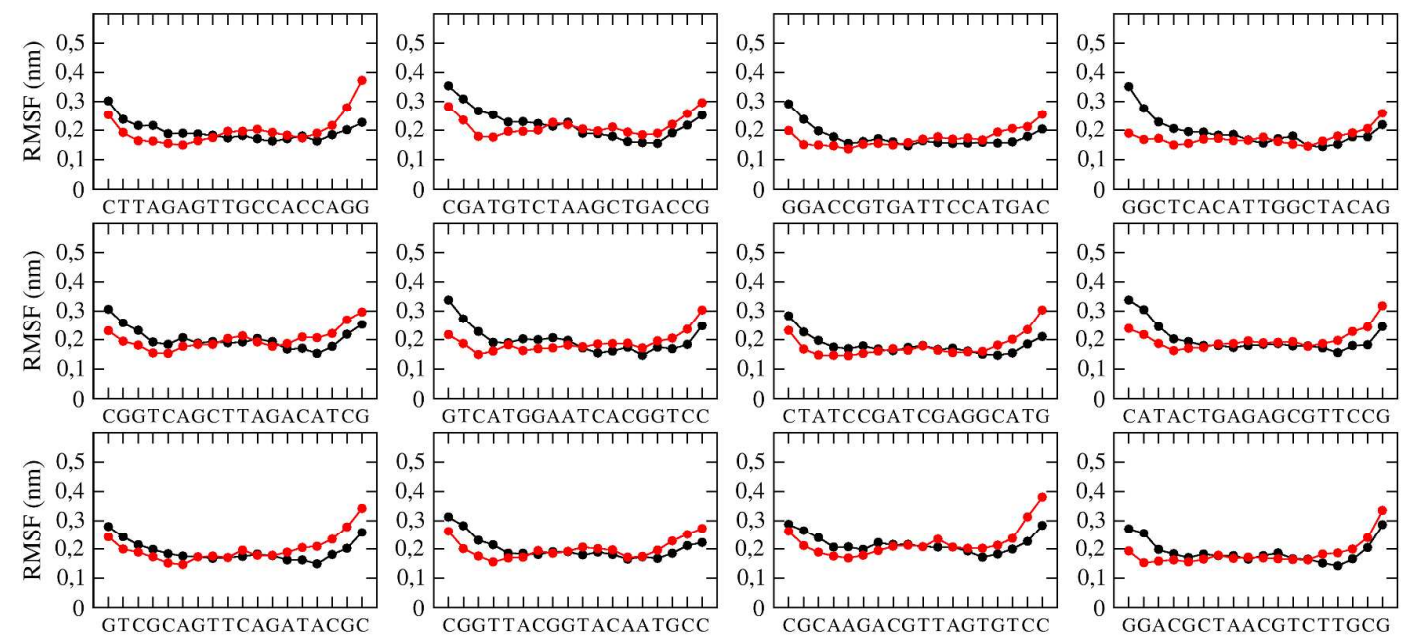

Figure S3B. Local RMSF values calculated for the double helices of the truncated cube cage. Black and red filled circles specify the two strands.
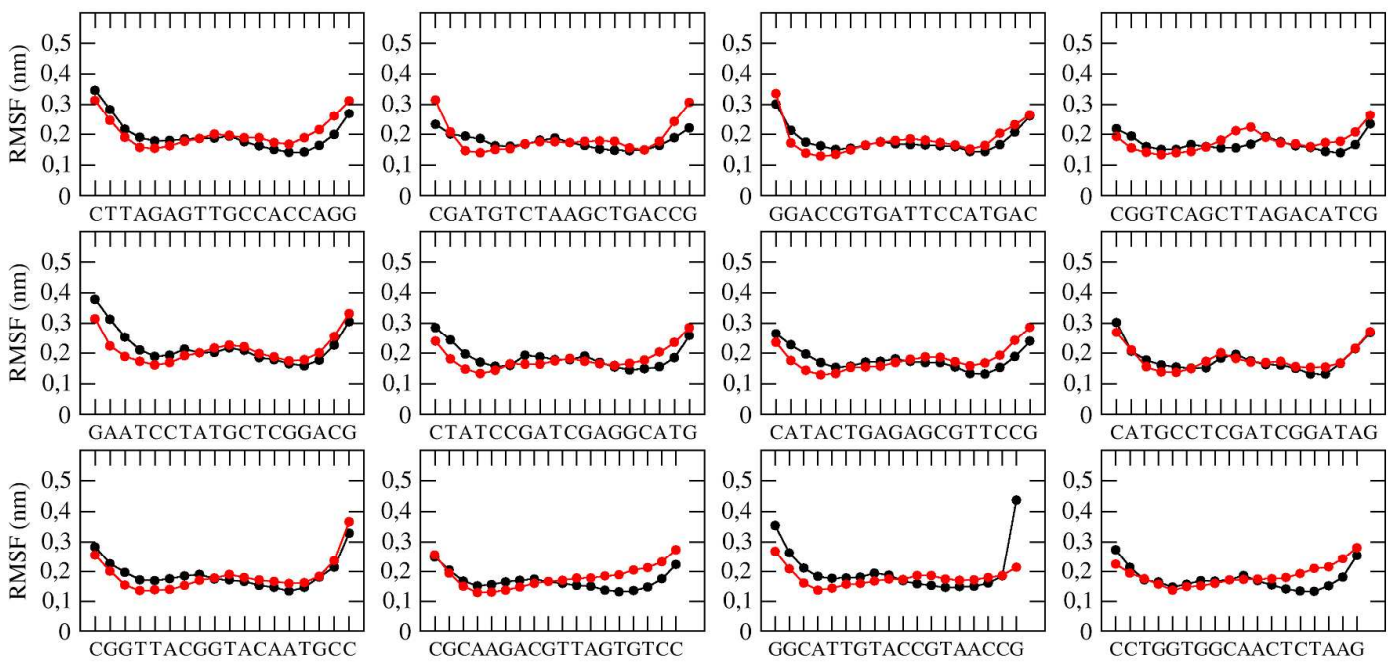

Figure S3C. Local RMSF values calculated for the double helices of the truncated octahedron cage. Black and red filled circles specify the two strands. 

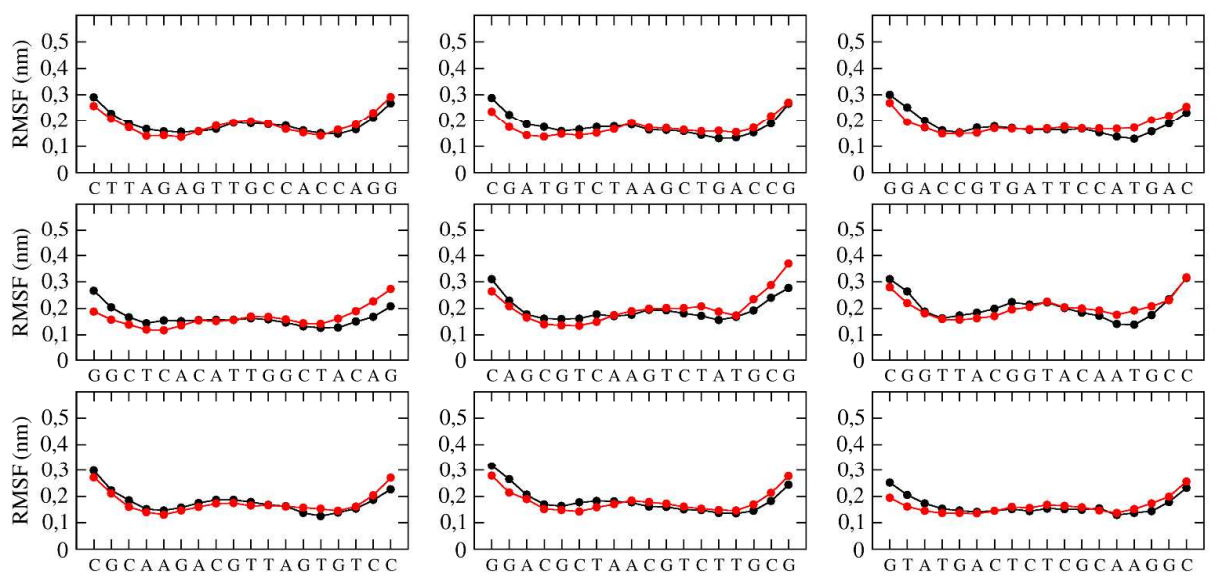

Figure S3D. Local RMSF values calculated for the double helices of the truncated triangular prism cage.

Black and red filled circles specify the two strands.

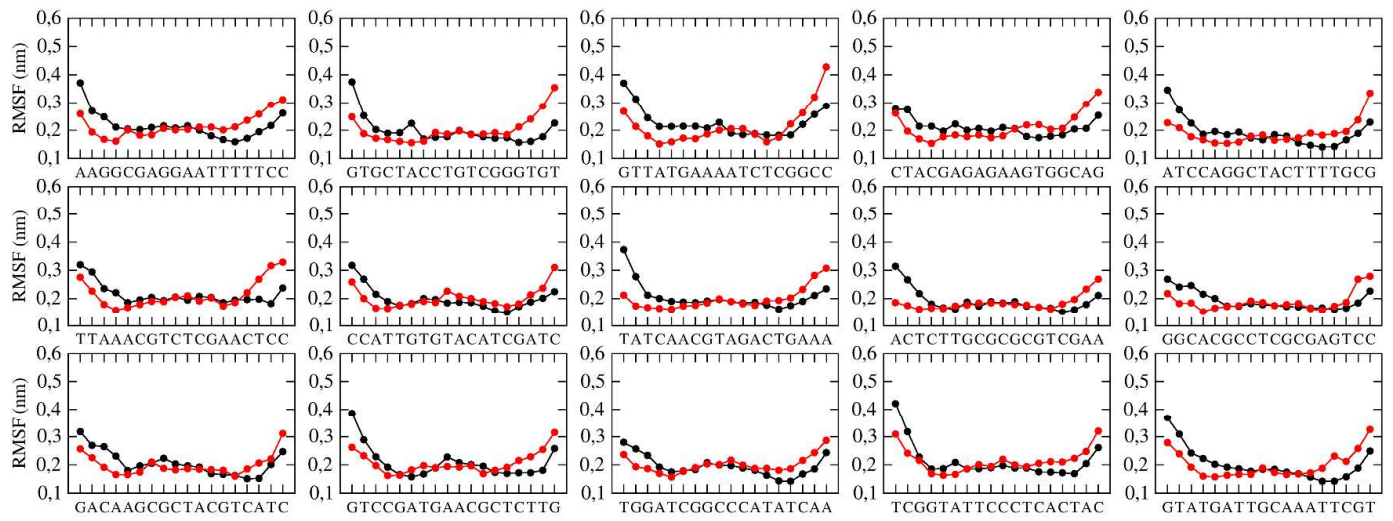

Figure S3E. Local RMSF values calculated for the double helices of the truncated pentagonal prism cage.

Black and red filled circles specify the two strands. 

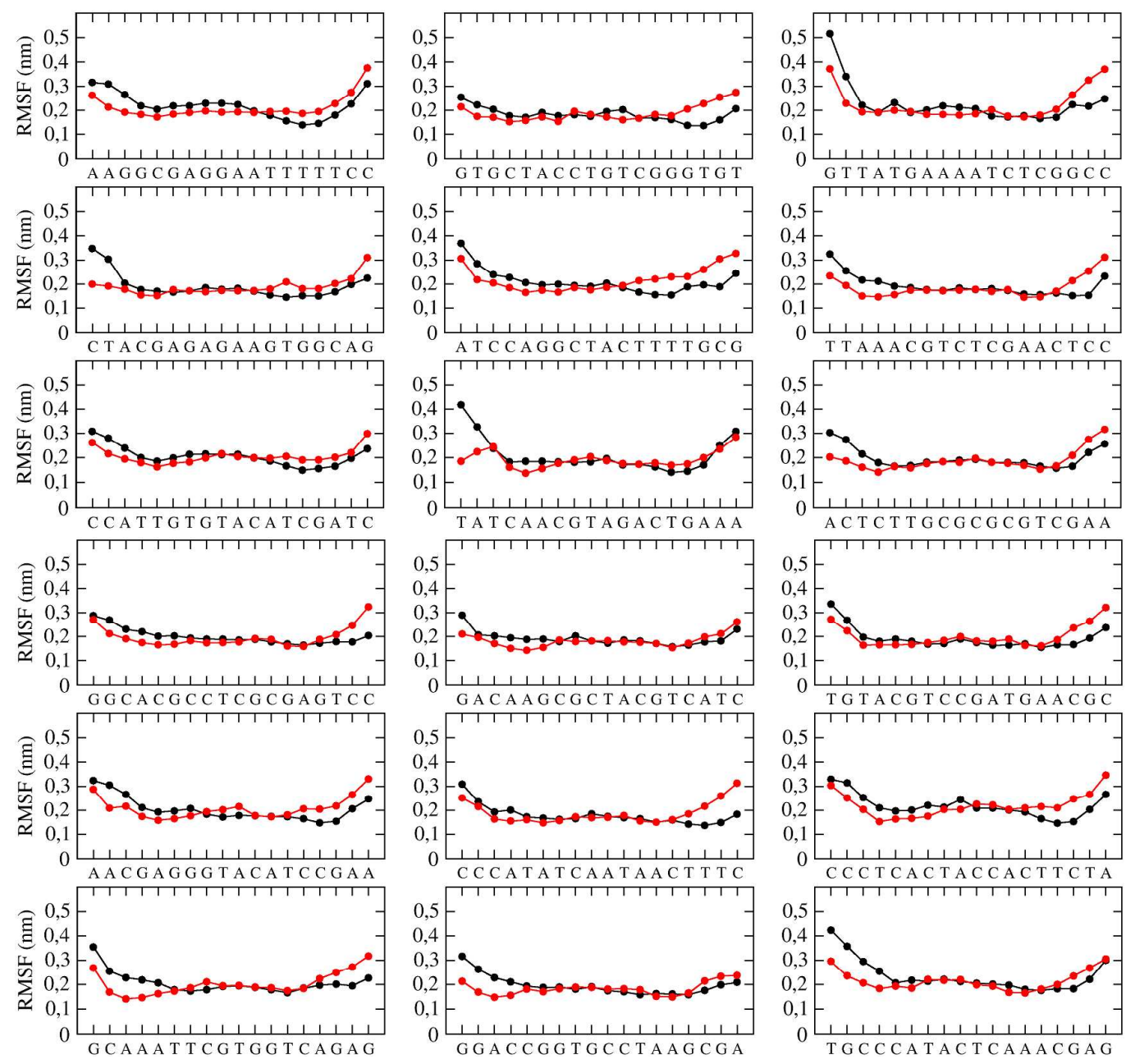

Figure S3F. Local RMSF values calculated for the double helices of the truncated hexagonal prism cage. Black and red filled circles specify the two strands. 

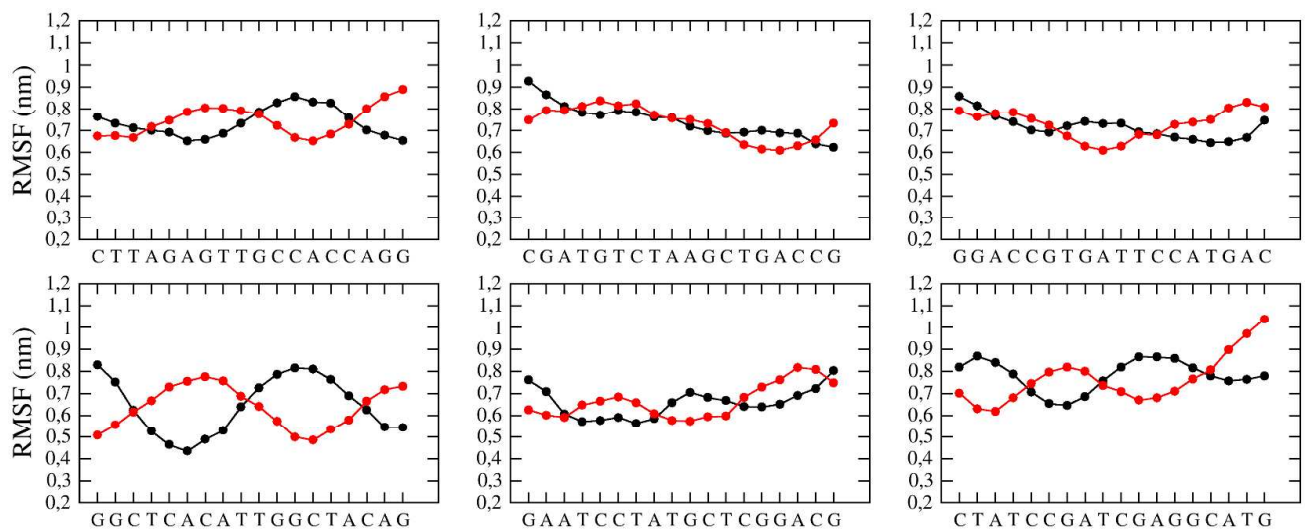

Figure S4A. Global RMSF values calculated for the double helices of the truncated tetrahedron cage. Black and red filled circles specify the two strands.
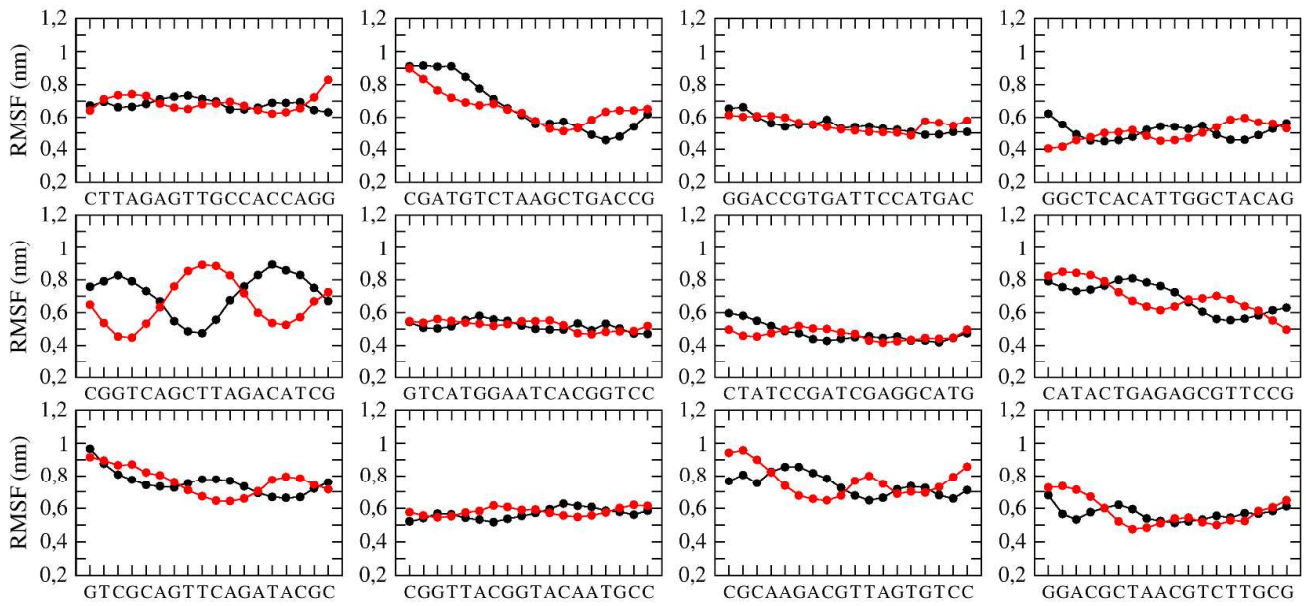

Figure S4B. Global RMSF values calculated for the double helices of the truncated cube cage. Black and red filled circles specify the two strands.
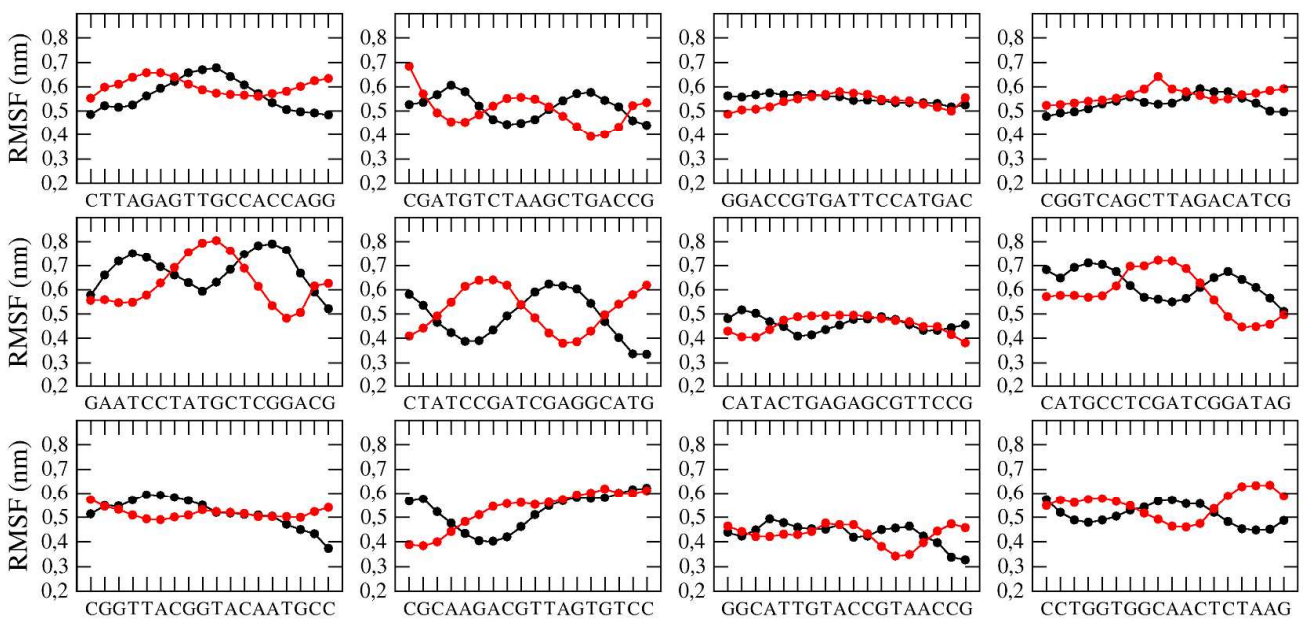

Figure S4C. Global RMSF values calculated for the double helices of the truncated octahedron cage. Black and red filled circles specify the two strands. 

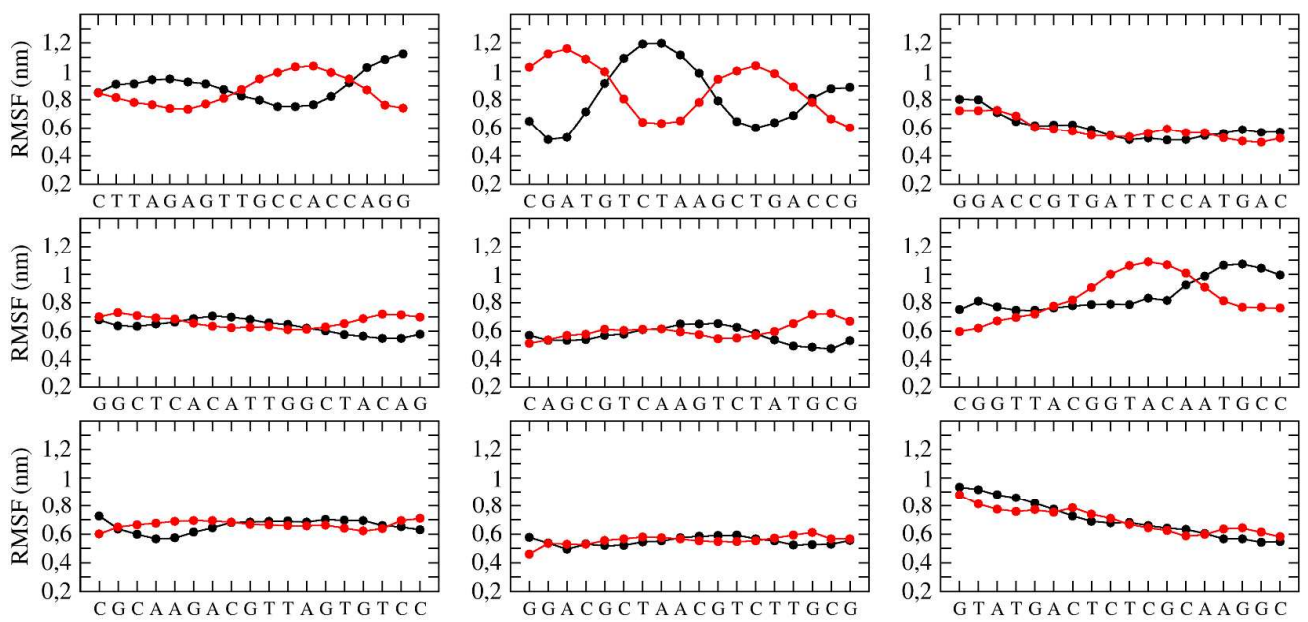

Figure S4D. Global RMSF values calculated for the double helices of the truncated triangular prism cage.

Black and red filled circles specify the two strands.
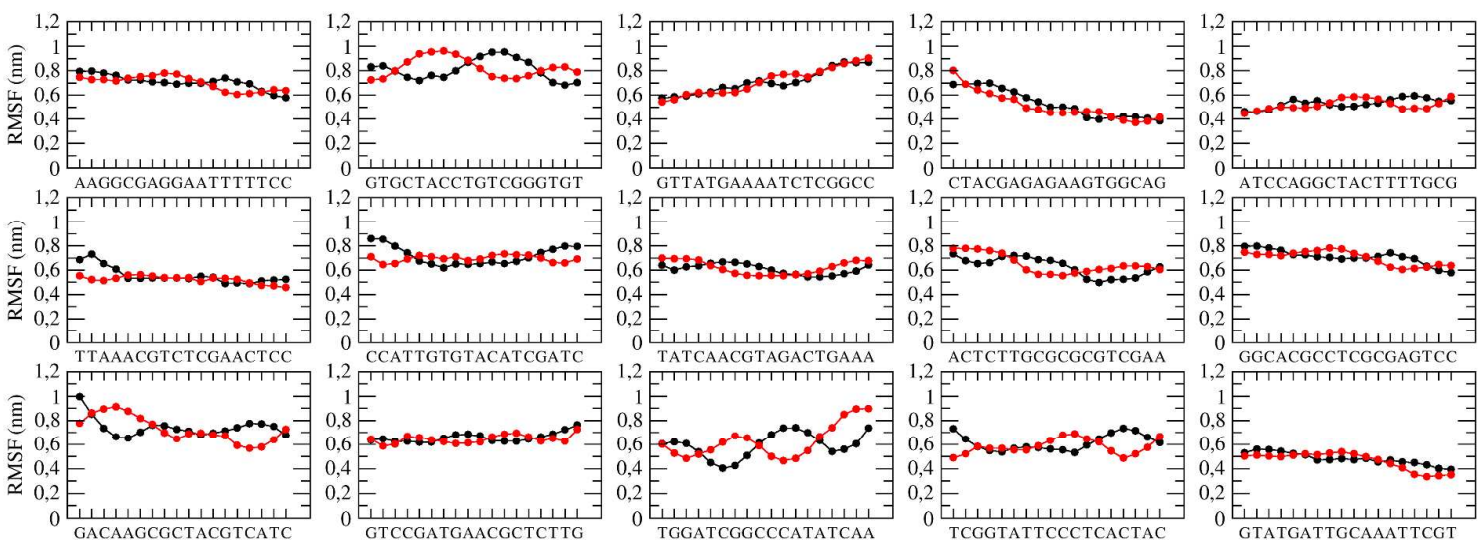

Figure S4E. Global RMSF values calculated for the double helices of the truncated pentagonal prism cage.

Black and red filled circles specify the two strands. 

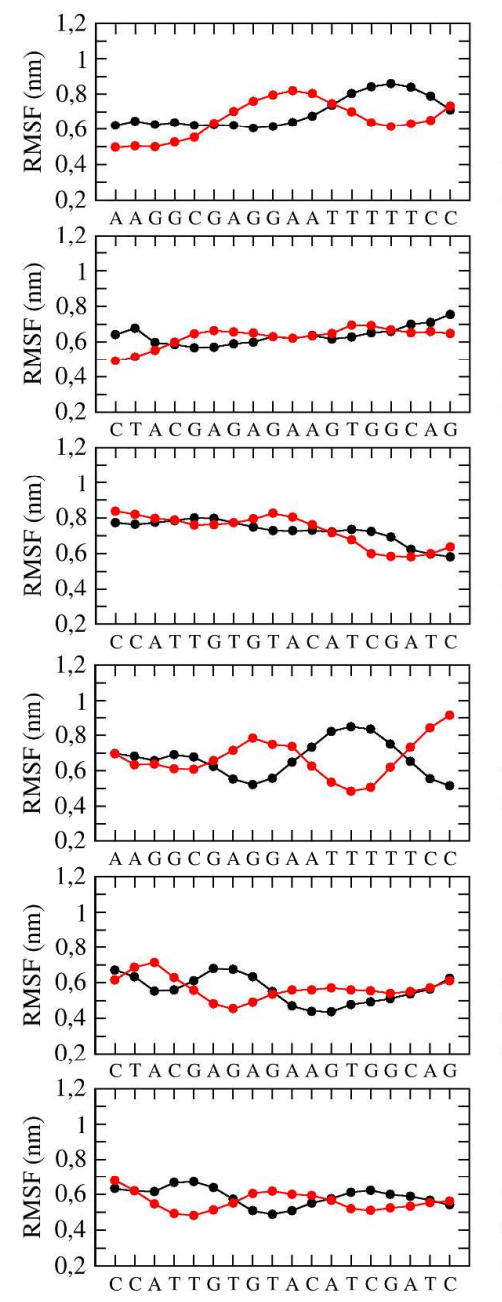

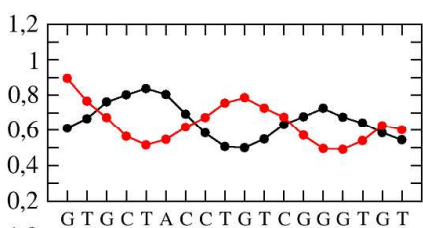

GTGCTACCTGTCGGGTGT
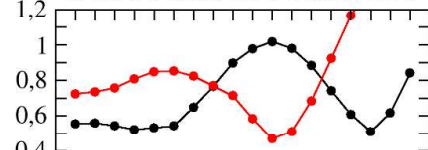

0,4

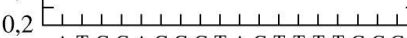
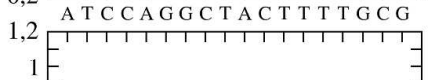

$0,8=$

0,4

$0,25,1,1+1,1,1,1,1,1,1,1$

TA T CAACGTAGACTGAAA

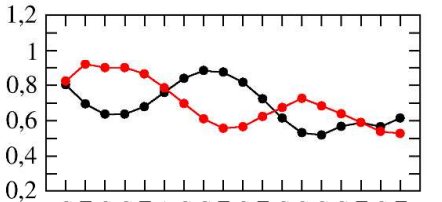

0,2 G
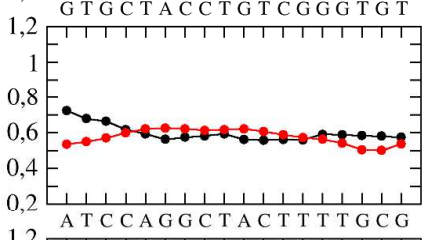

A T C CAGGCT A C T T T T G C G

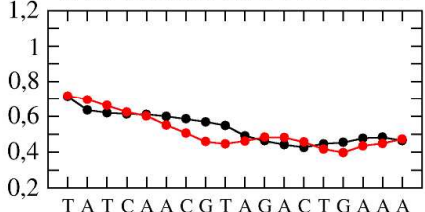

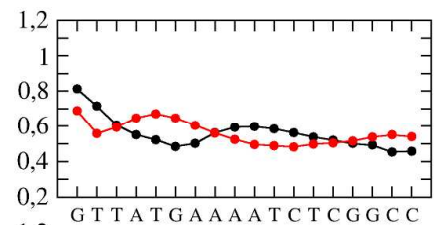

2 G T T A T GAAAA T C T CGGCC

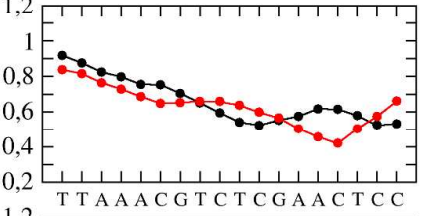

T TAAACG T C T C GAAC T C C
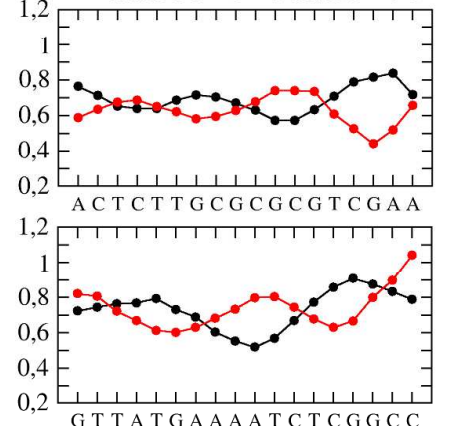

GT TA TGAAAATCTCGGCC

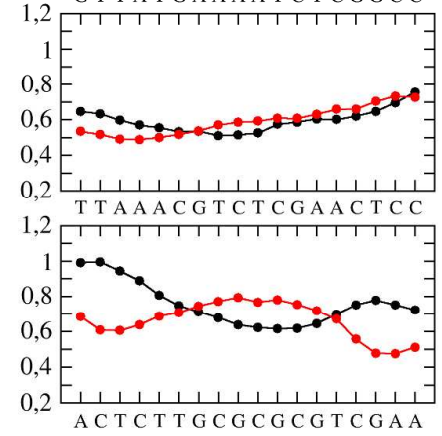

Figure S4F. Global RMSF values calculated for the double helices of the truncated hexagonal prism cage. Black and red filled circles specify the two strands. 


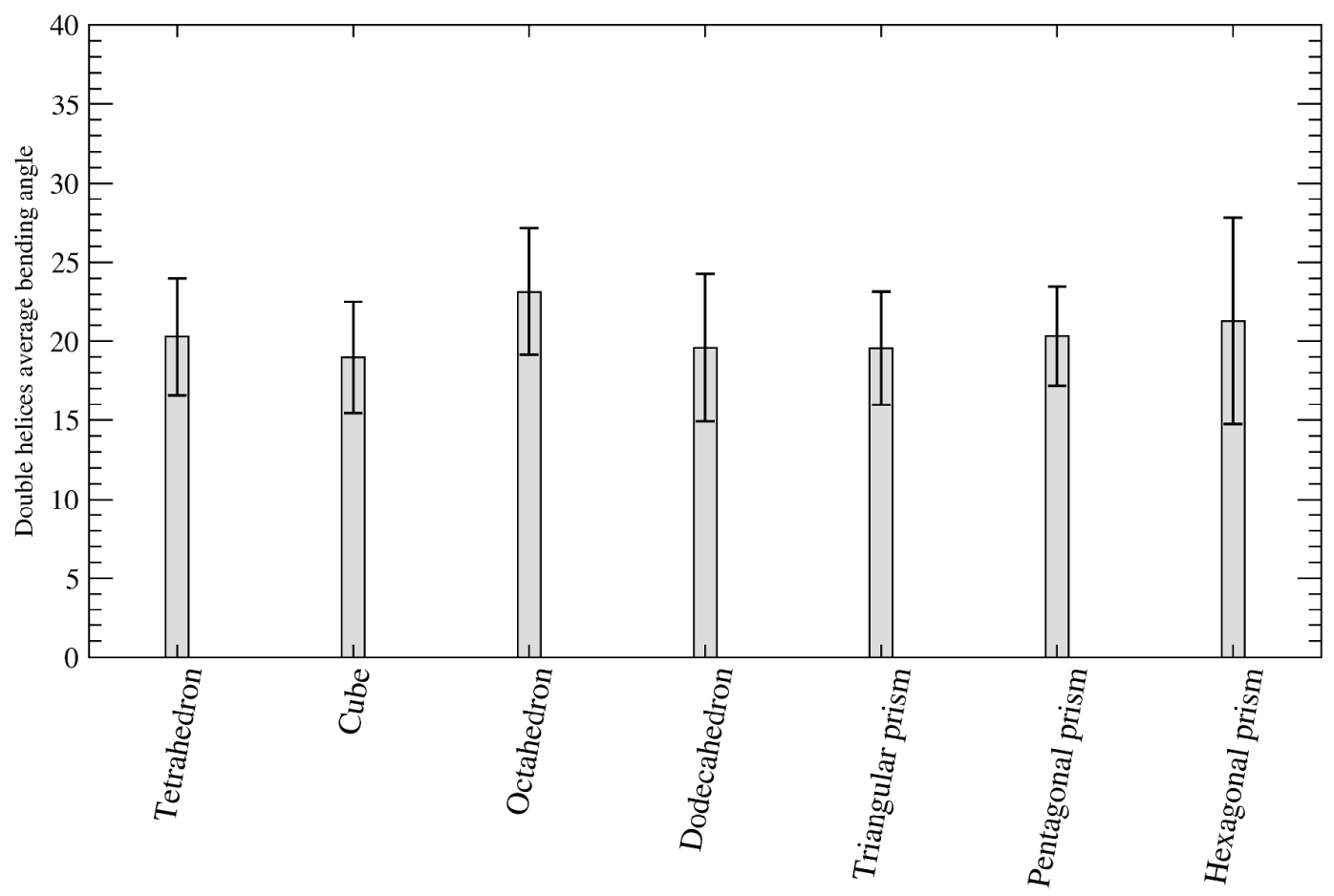

Figure S5. Histogram indicating the average bending angle characterizing the DNA double helices of the seven different cages.

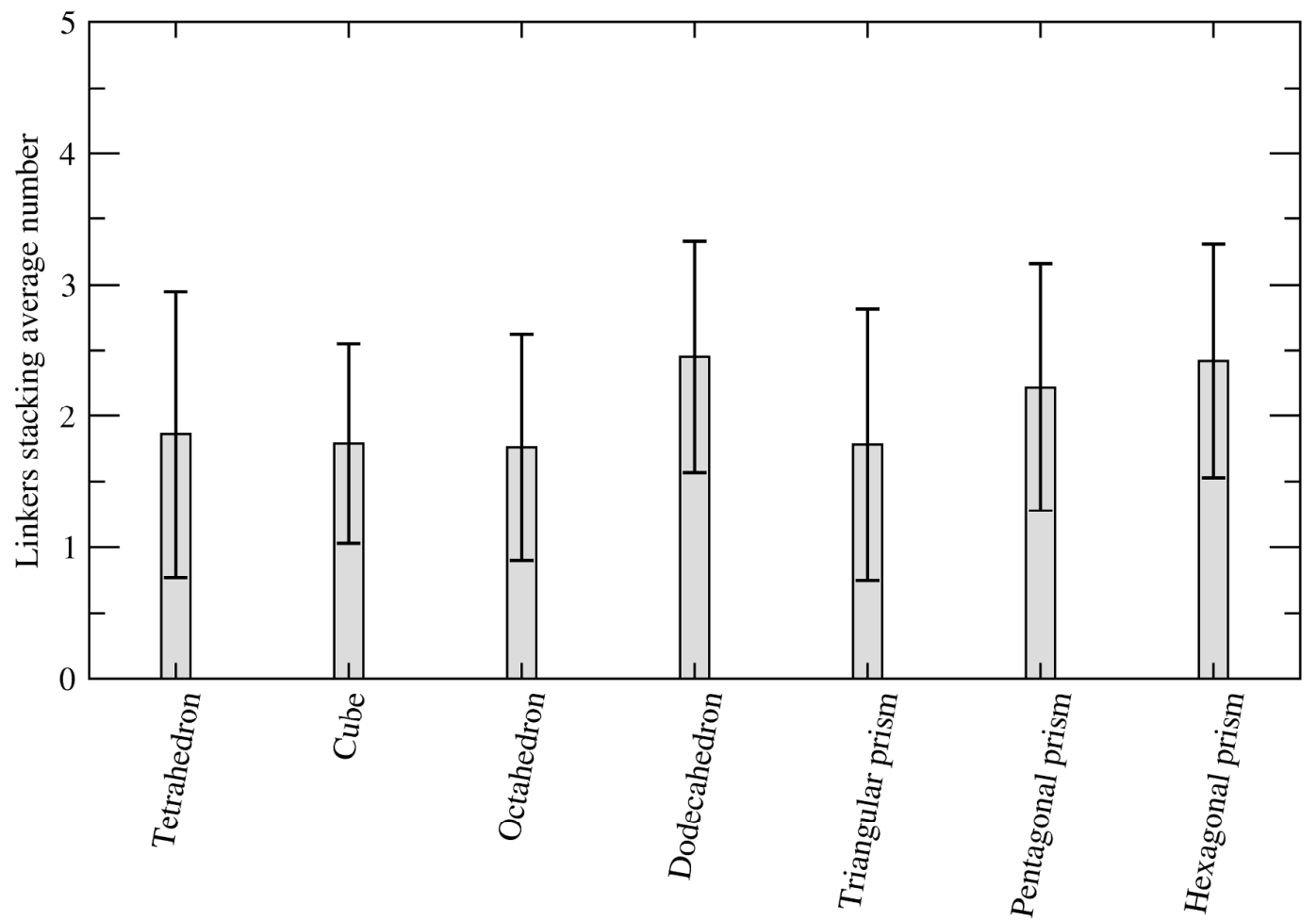

Figure S6. Histogram indicating the average number of base stackings established in the DNA single strand linkers of the seven different cages. 


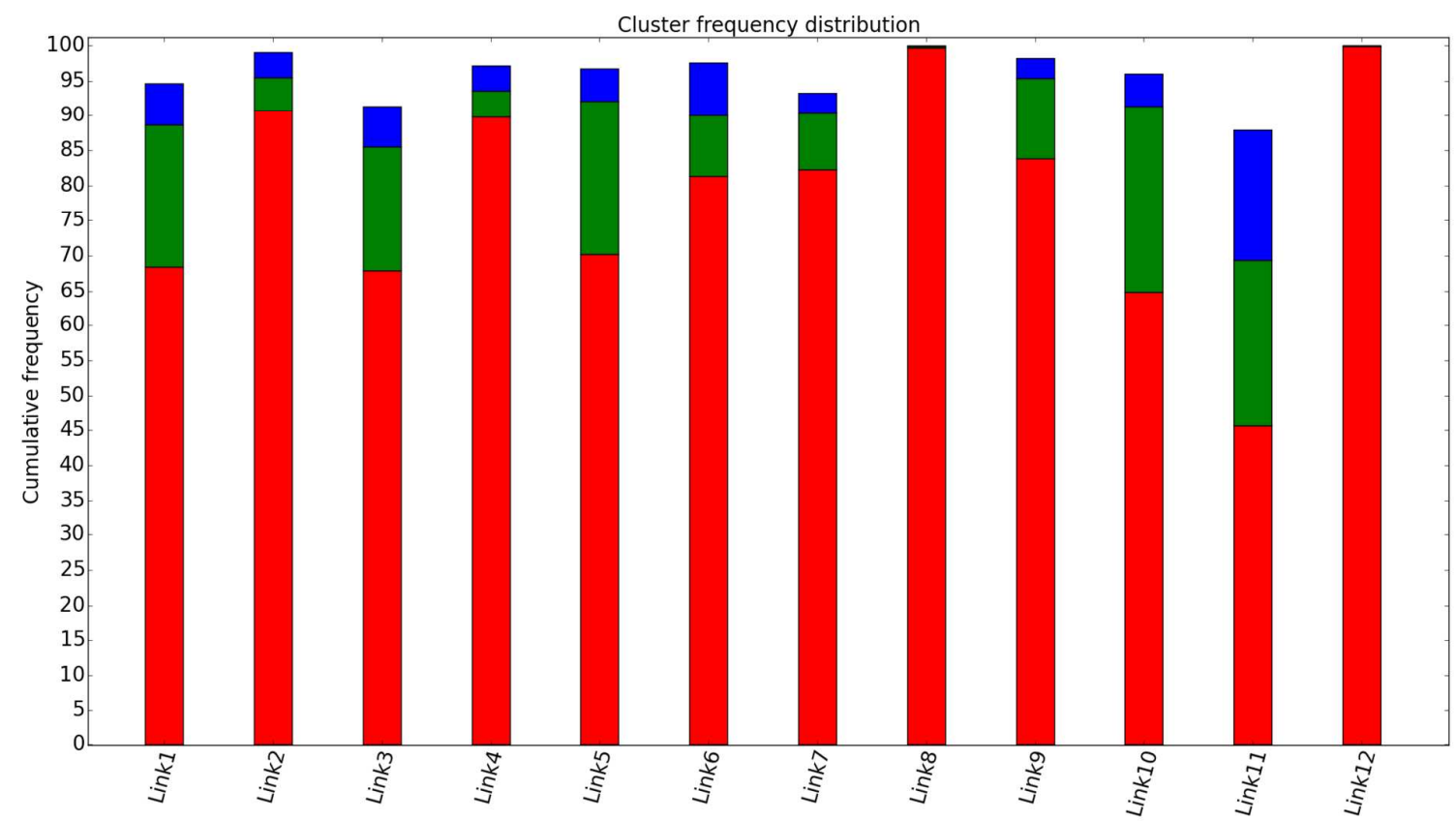

Figure S7A. Results of the linker cluster analysis for the truncated tetrahedron cage. Each linker is represented by a stacked bar in which the percentage of the three most populated clusters is indicated. The first cluster is represented in red while the second and third ones are represented in green and blue, respectively.

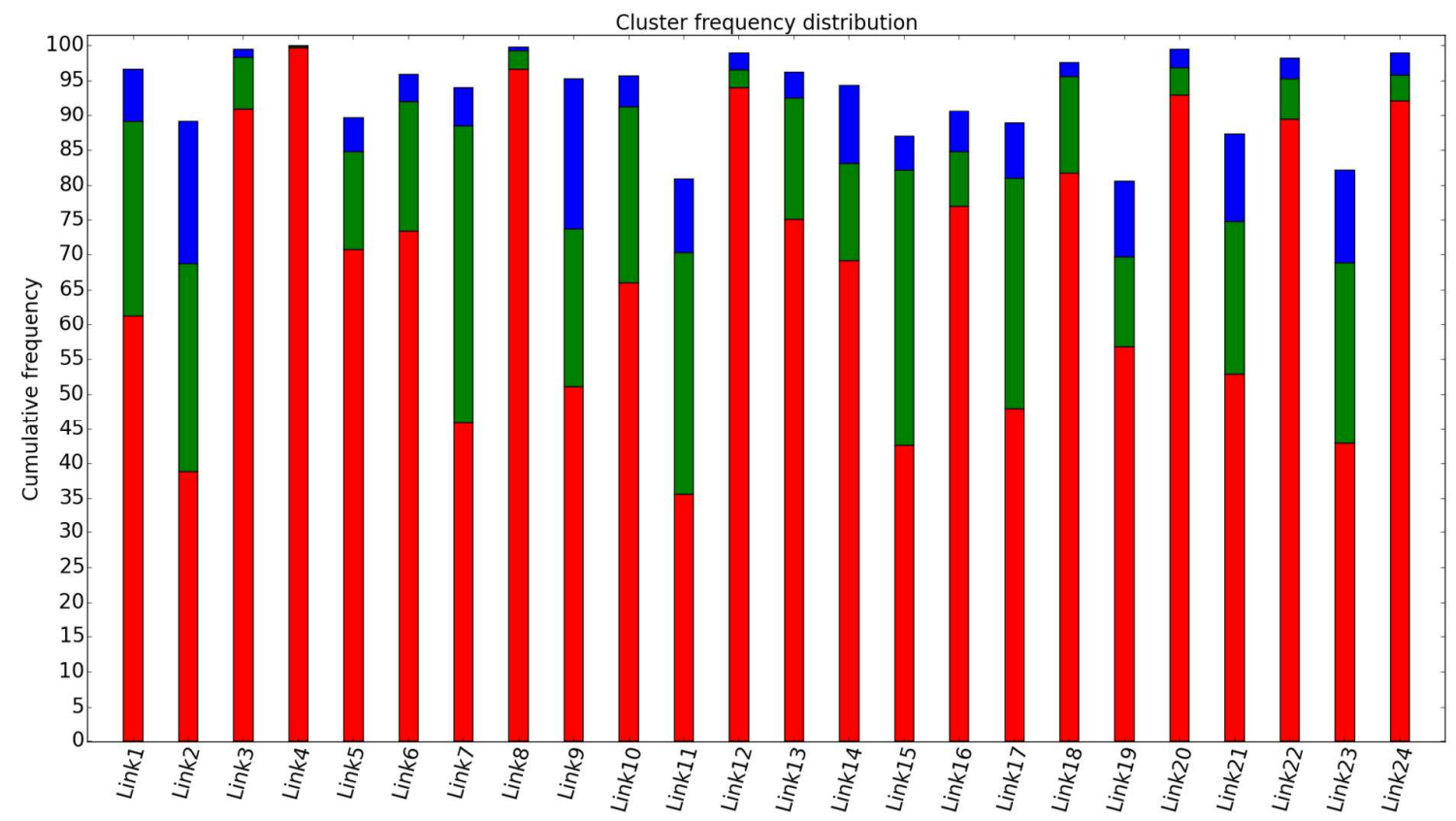

Figure S7B. Results of the linker cluster analysis for the truncated cube cage. Each linker is represented by a stacked bar in which the percentage of the three most populated clusters is indicated. The first cluster is represented in red while the second and third ones are represented in green and blue, respectively. 


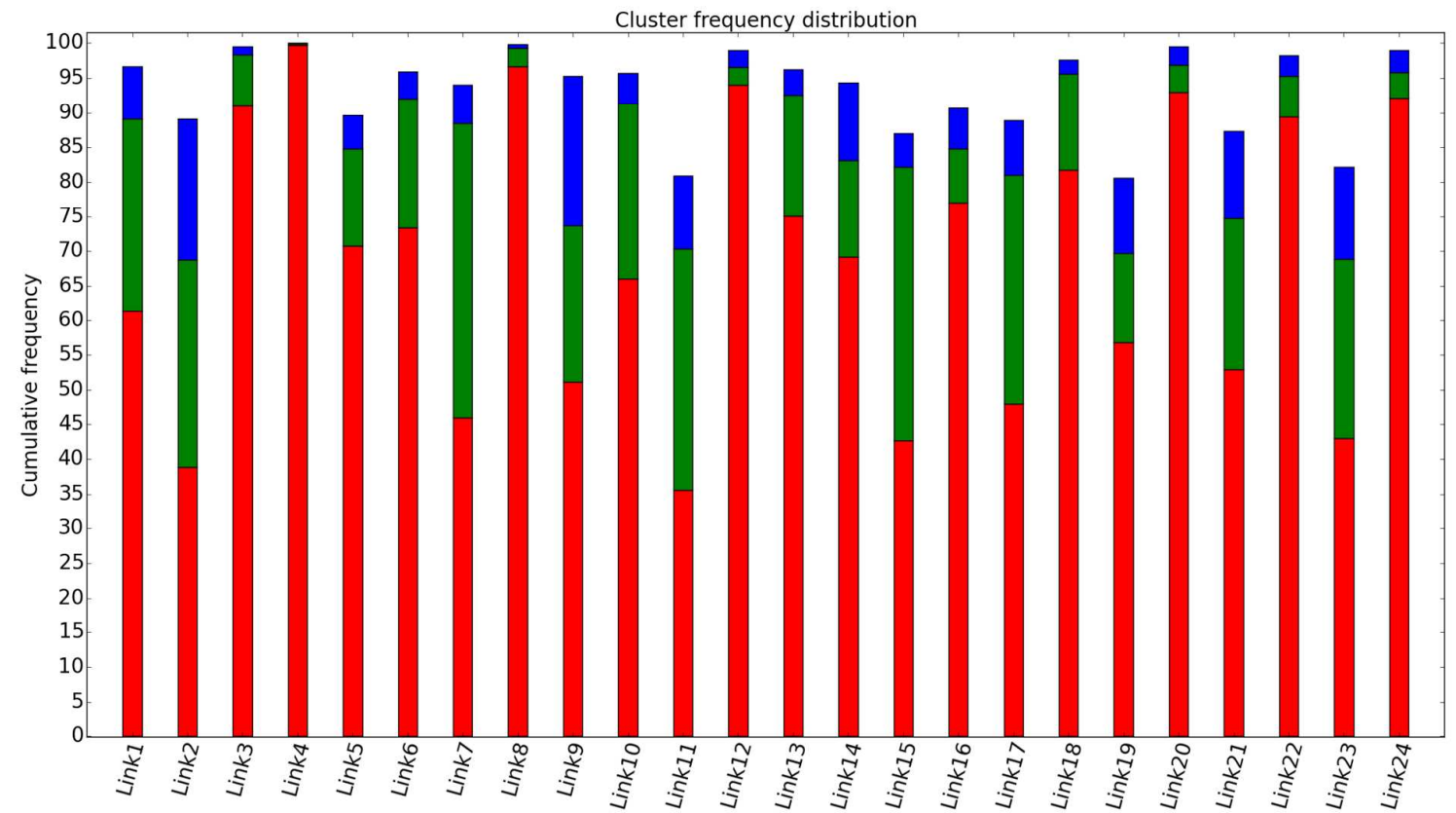

Figure S7C. Results of the linker cluster analysis for the truncated octahedron cage. Each linker is represented by a stacked bar in which the percentage of the three most populated clusters is indicated. The first cluster is represented in red while the second and third ones are represented in green and blue, respectively.

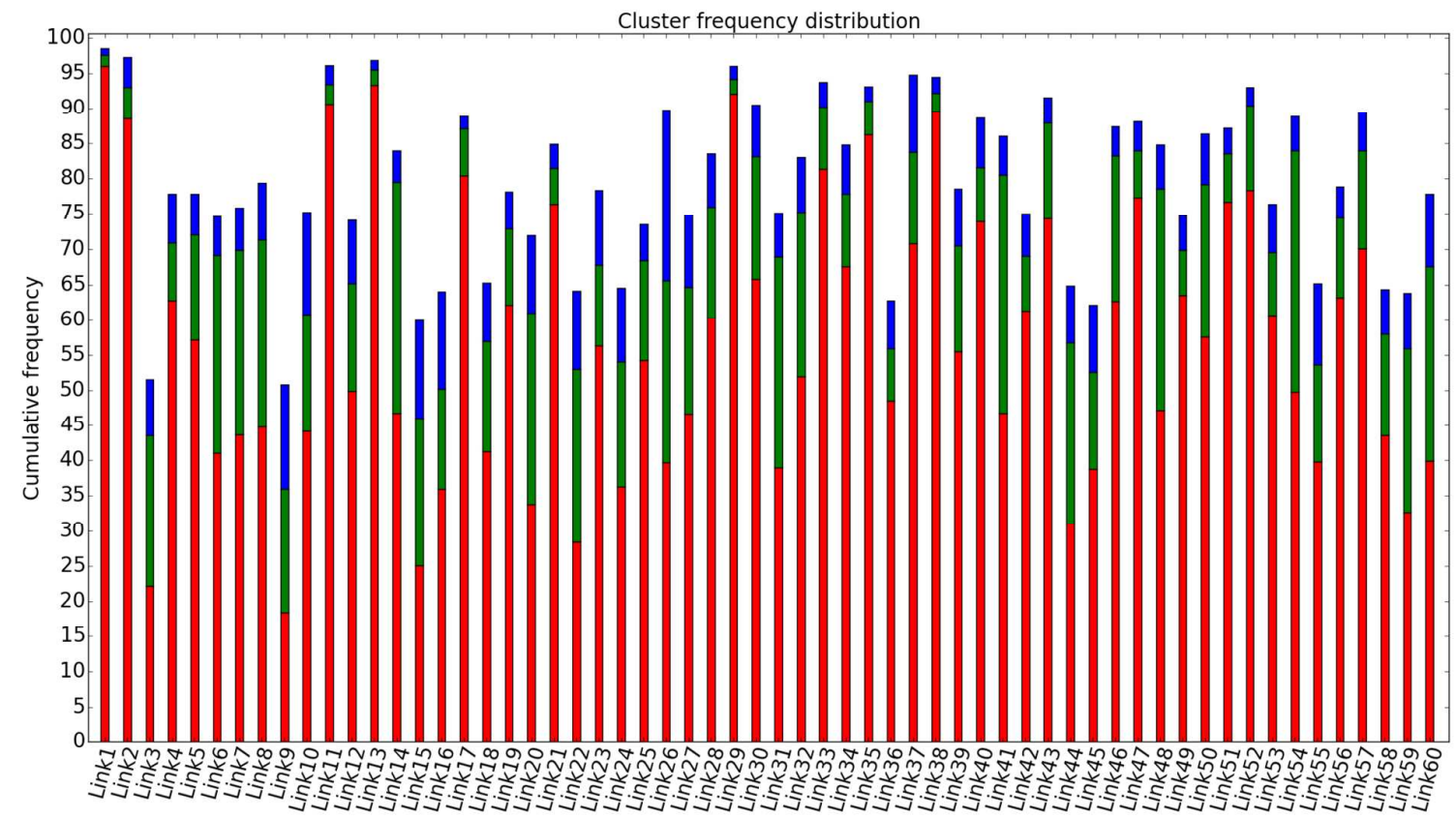

Figure S7D. Results of the linker cluster analysis for the truncated dodecahedron cage. Each linker is represented by a stacked bar in which the percentage of the three most populated clusters is indicated. The first cluster is represented in red while the second and third ones are represented in green and blue, respectively. 


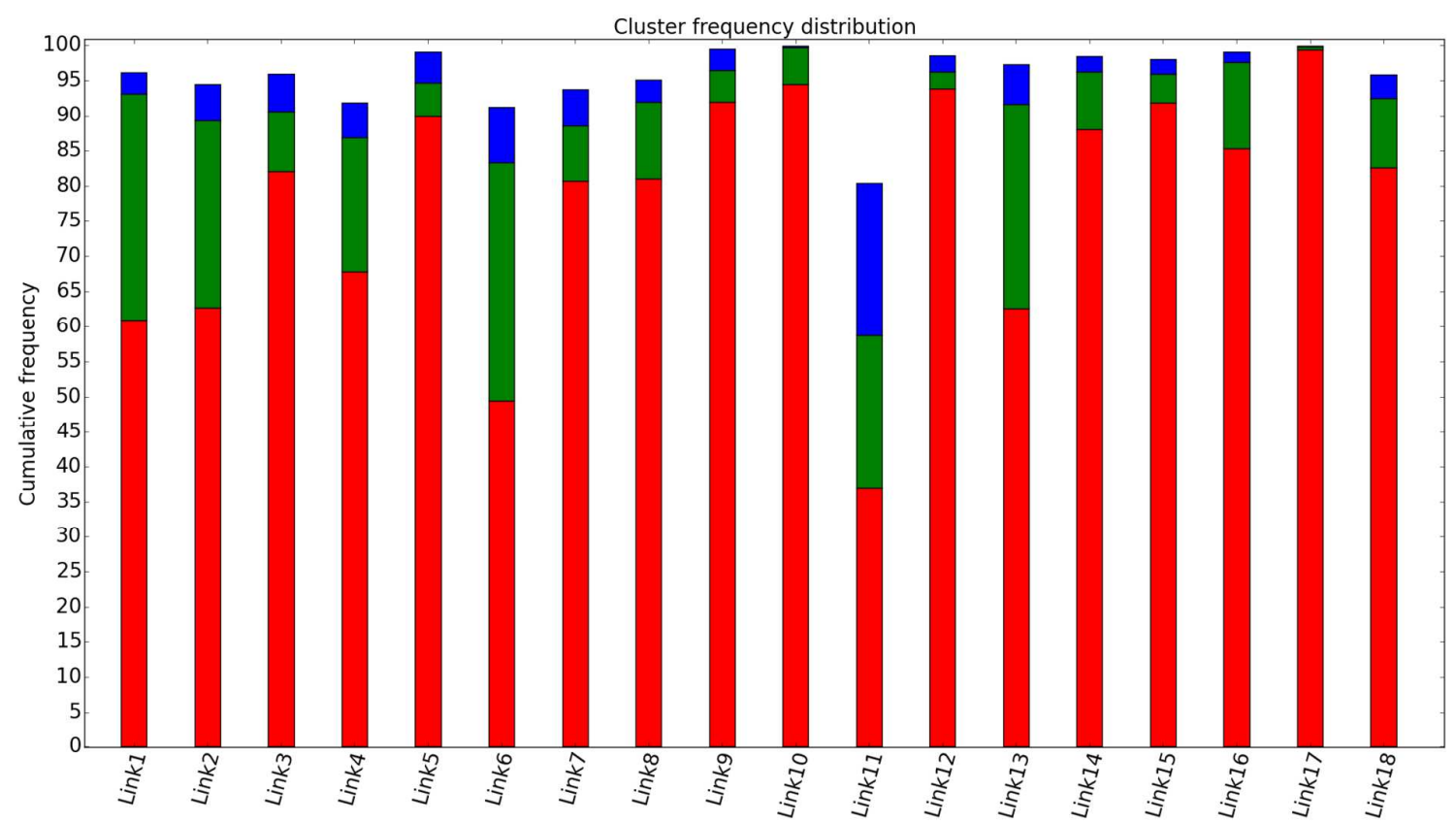

Figure S7E. Results of the linker cluster analysis for the truncated triangular prism cage. Each linker is represented by a stacked bar in which the percentage of the three most populated clusters is indicated. The first cluster is represented in red while the second and third ones are represented in green and blue, respectively.

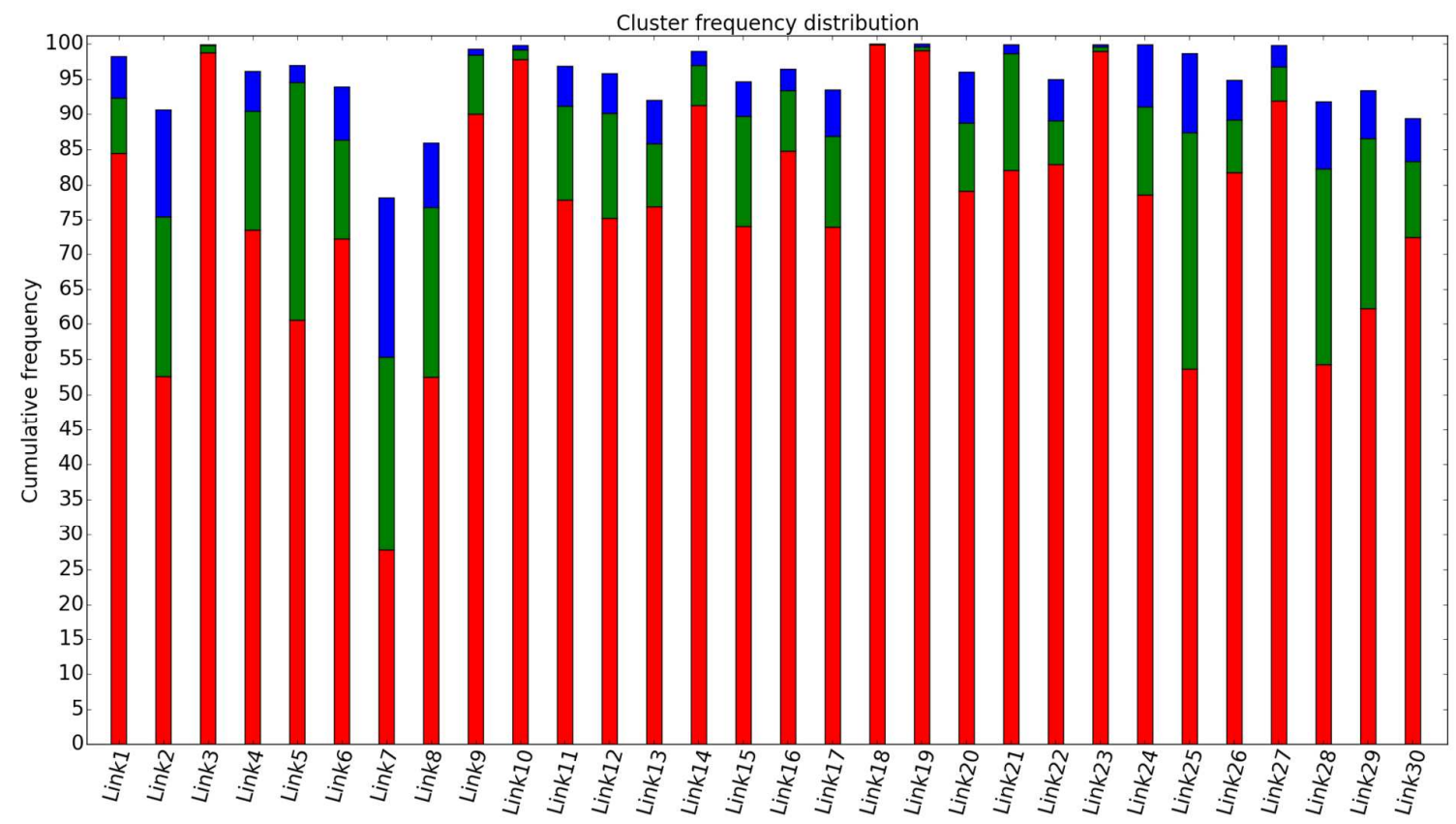

Figure S7F. Results of the linker cluster analysis for the truncated pentagonal prism cage. Each linker is represented by a stacked bar in which the percentage of the three most populated clusters is indicated. The first cluster is represented in red while the second and third ones are represented in green and blue, respectively. 


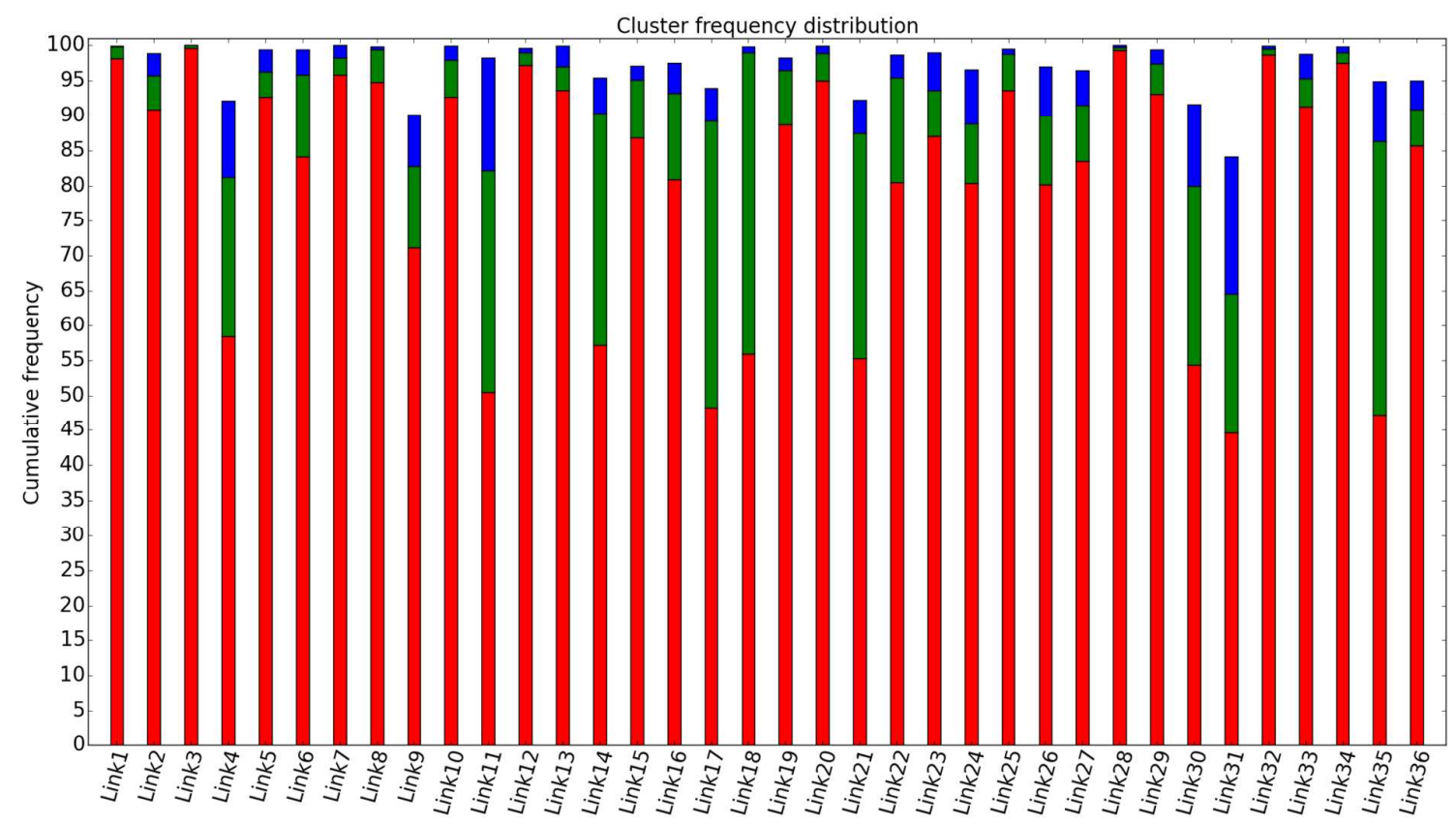

Figure S7F. Results of the linker cluster analysis for the truncated hexagonal prism cage. Each linker is represented by a stacked bar in which the percentage of the three most populated clusters is indicated. The first cluster is represented in red while the second and third ones are represented in green and blue, respectively. 